\title{
Movement Properties of Elderly Pedestrians in the Bottleneck Flow With Different Compositions
}

\author{
Xiangxia Ren · Jun Zhang · Weiguo Song \\ State Key Laboratory of Fire Science, University of Science and Technology of China, Hefei, \\ China, \\ E-mail: renxia@mail.ustc.edu.cn, junz@ustc.edu.cn,wgsong@ustc.edu.cn
}

Received: 13 August 2021 / Last revision received: 6 October 2021 / Accepted: 2 November 2021 DOI: $10.17815 / C D .2021 .121$

\begin{abstract}
With the development of aging society, the proportion of the old population is ascending continually. The elders facing the challenge of impaired mobility and decreasing flexibility will affect the dynamic characteristics of pedestrian flow. While the study of the evacuation dynamic mainly focuses on the young for the limited experimental conditions. The movement of elderly pedestrians are seldom investigated. However, the comprehensive understanding of the influence of the elders on the pedestrian flow is essential for facility design and evacuation management. Therefore, a series of comparative experiments between the separate elderly group, separate young group and the mixed group (38 elders and 63 young) are carried out to study the movement properties of elderly pedestrians in the bottleneck flow with different compositions quantitatively. The mixture of the elderly and the young makes the efficiency decrease significantly. In the mixed group, the speed of the young pedestrians is lower than that of the elders for the young slow down to avoid collisions. The young kept a certain distance from the elders for the sake of safety, so that the high-density profiles of the mixed group present as long strips. In addition, pedestrians in the mixed group have longer time lapse than that in the separate groups when passing the bottleneck. In the separate groups, the spatial distribution of the nearest neighbour presents as a uniform circle. The spatial distance between the elderly $(0.46 \mathrm{~m})$ is bigger than that between the young $(0.39 \mathrm{~m})$. While there is no obvious difference in the mixed group. The results can be used in pedestrian modelling, facilities designing and evacuation management for the population with old people.
\end{abstract}

Keywords Elderly group · young group $\cdot$ mixed group $\cdot$ bottleneck flow $\cdot$ density $\cdot$ speed 


\section{Introduction}

Comprehensive knowledge on pedestrian dynamics is of key importance of facility design and crowd management, especially under some emergencies, such as fire accidents and earthquake. The studies on human safety and pedestrian dynamics are growing rapidly in recent years [1,2]. Population aging is one of the most significant trends of the $21 \mathrm{st}$ century. Changes in crowd composition can have an impact on pedestrian dynamics and transportation systems for the elderly are vulnerable [3]. In response to the change of population structure, more attention should be paid to the study of the passage efficiency and the safety of pedestrians in public facilities. However, most of the previous studies focused on the young adults with single composition considering the operability of the control experiments. Only few works were made to consider the influence of elderly pedestrians and crowd compositions on pedestrian dynamics $[4,5]$. The movement properties of elderly pedestrians through bottlenecks are seldom investigated. In addition, the mixing effect of pedestrians of different age remains to be explored.

Bottleneck structure is a key position of traffic facilities that restricts the efficiency of pedestrian passage [6]. It has been paid much attention on the dynamics properties of pedestrian flow through the bottleneck [7], including controlled experiments and simulation model. Flow rate is an important consideration for the efficiency of pedestrians crossing bottlenecks. It has been found in the previous studies [8,9] that the flow rate increases linearly with the increase of exit width. But the situation is different when the exit location is in the corner [10]. The increasing age of pedestrians has a negative impact on the magnitude and growth slope of the flow rate [11,12]. While the influence of mixture of pedestrians with different ages has not been studied. Speed and density are basic parameters for describing pedestrian dynamics. To observe the relation between speed and density, the fundamental diagram [13] has been widely studied. It can be observed in the previous study [11] that the separately elderly group has lower speed and density than the separately young one. In addition, Voronoi diagram method [14] usually be used to show the density files. The outline shape and peak value of the density profiles can reflect the congestion situation of the crowd. To study the mechanisms of efficiency and congestion, time headway between two continuous pedestrians [15] and spatial distribution [16] of the nearest neighbour can show the pedestrian dynamic through temporal and spatial aspects respectively. However, the previous studies mainly focus on the crowd with pedestrians within a certain age range. The influence of the mixture of young and elderly pedestrians on the dynamic is ambiguous.

In daily life, the mixed traffic flow of elderly and young people is common, especially in places where old people gathering, such as hospital and nursing homes. The facilities design and crowd management of these places require further comparison studies on dynamics of the mixing crowd. The remaining of this paper are arranged as follows: Section 2 introduces the experimental setup and Section 3 exhibits the data analysis method. In Section 4, we show the results and discussions, including flow rate, speed differences, density profiles, time headway and spatial distribution. Finally, Section 5 summarizes the paper and makes the conclusions. 


\section{Experiment setup}

The experiments are carried out in the University of Science and Technology in Hefei, Anhui province. Three series of the set up are considered to observe the elderly movement in different compositions, including the separated elderly group, separated young group and mixed group. The young are students recruited from the university and the elders are recruited from a senior centre in Hefei. The mean age of the elderly group is 74.5 ranging from 60 to 86 . The young group has an average age of 23.0 ranging from 17 to 28. Only elderly (young) participants in the separated elderly (young) group. While in the mixed group, the ratio of the elders to the young is around 1:1.5. Considering the limited physical strength of the elderly, we follow the wishes of each elder to participate in each run and arranged adequate rest to ensure the safety, which also led to the inconsistency in the number of the elderly in each run. The details of the experimental set up can be found in Tab. 1.

The experimental scenario is a built room surrounding by $1.8 \mathrm{~m}$ height boards. The width of the exit varies from $0.6 \mathrm{~m}$ to $1.6 \mathrm{~m}$ at an interval of $0.2 \mathrm{~m}$. Fig. 1 shows the schematic illustration of the scenario and a snapshot of the experiment. At the beginning, the participants are asked to wait in the waiting area in queue. In the mixed group, the elders alternate with the young in rows. See Fig. 1(b), the orange hats are for the elders and the red ones are for the young. When the experiment starts, all of the participants are required to walk through the built room as soon as possible.

The process of the experiments is recorded by two cameras fixed at the height about 10 $\mathrm{m}$. The software PeTrack [17] is used to process video information. Two calibrations are carried out before and after the experiments according to the software user manual. The trajectories of each participant can be extracted through the video by tracking the brightly colored hats. Fig. 1(b) shows the trajectory tracking results of five elderly pedestrians. According to the trajectory data, the parameters such as pedestrian velocity, density and flow rate can be further calculated and analysed.

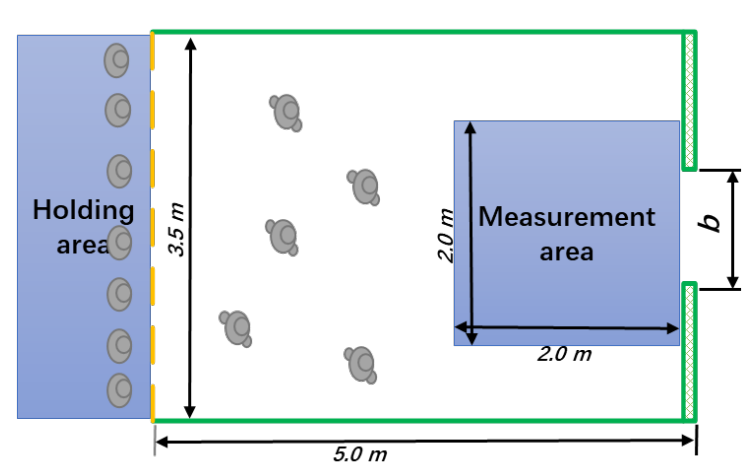

(a)

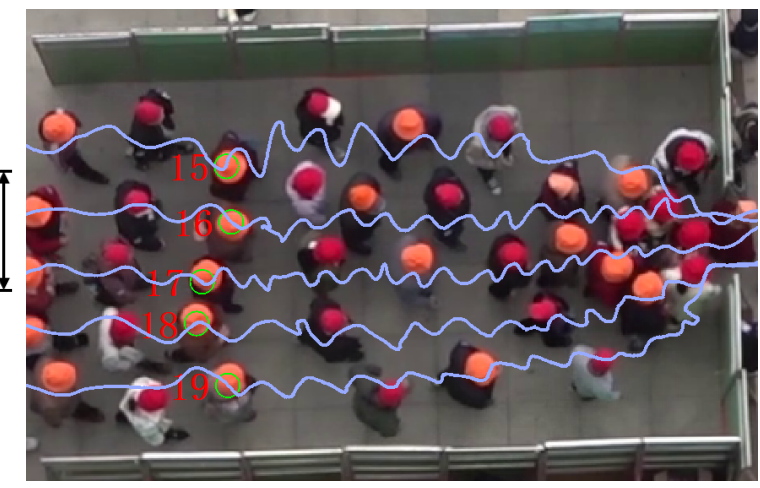

(b)

Figure 1 (a) The schematic illustration of the scenario and (b) a snapshot of the experiment with trajectories 


\begin{tabular}{|c|c|c|c|c|}
\hline \multirow[t]{2}{*}{ condition } & \multirow[t]{2}{*}{ elderly group } & \multirow[t]{2}{*}{ young group } & \multicolumn{2}{|c|}{ mixed group } \\
\hline & & & number of elders & number of young \\
\hline$b=0.6 \mathrm{~m}$ & 65 & 84 & 39 & 63 \\
\hline$b=0.8 \mathrm{~m}$ & 44 & 84 & 39 & 63 \\
\hline$b=1.0 \mathrm{~m}$ & 63 & 84 & 38 & 63 \\
\hline$b=1.2 \mathrm{~m}$ & 45 & 84 & 38 & 63 \\
\hline$b=1.4 \mathrm{~m}$ & 34 & 84 & 37 & 63 \\
\hline$b=1.6 \mathrm{~m}$ & 35 & 84 & 38 & 63 \\
\hline
\end{tabular}

Table 1 The details of pedestrian compositions of different runs in our experiment

\section{Data analysis method}

The flow rate $\mathrm{J}$ and flow $\mathrm{J}_{S}$ are defined as below:

$$
\begin{gathered}
J=\frac{N_{t}}{t} \\
J_{s}=\frac{N_{t}}{t \cdot b}
\end{gathered}
$$

where $N_{t}$ is the cumulative number of passengers passing through the exit at time $t$ and $b$ is width of the exit.

The instantaneous velocity $v_{i}(t)$ of pedestrian $i$ in time $t$ is defined as below:

$$
v_{i}(t)=\frac{\sqrt{\left[x_{i}(t+\Delta t / 2)-x_{i}(t-\Delta t / 2)\right]^{2}+\left[y_{i}(t+\Delta t / 2)-y_{i}(t-\Delta t / 2)\right]^{2}}}{\Delta t}
$$

where $x_{i}(t) y_{i}(t)$ is the $x$ and $y$ coordinate of pedestrian $i$ at time $t$ and $\Delta t=0.4 \mathrm{~s}$ is used in our analysis.

The average density $\rho$ and velocity $v$ for the measurement area based on the Voronoi method [14] are calculated by Eq. 4 and Eq. 5:

$$
\begin{gathered}
\rho=\frac{\iint \rho_{x y} d_{x} d_{y}}{b \cdot \Delta x} \\
v=\frac{\iint v_{x y} d_{x} d_{y}}{b \cdot \Delta x}
\end{gathered}
$$

where $\rho_{x y}=1 / \mathrm{A}_{i}$ and $\mathrm{v}_{x y}=\mathrm{v}_{i}(\mathrm{t})$ if $(\mathrm{x}, \mathrm{y}) \in \mathrm{A}_{i}$ for $\mathrm{A}_{i}$ is the Voronoi cell area for pedestrian $i$ and $\mathrm{v}_{i}$ is the instantaneous velocity for pedestrian $i$.

\section{Results and discussions}

\subsection{Flow rate}

The flow rate is defined as the number of pedestrians passing through the exit in unit time. The relation between the flow rate $\mathrm{J}$ and the exit width $b$ is studied widely. Fig. 2(a) 
compares the flow-width relations of the separate elderly group, separate young group and the mixed group and the linear fitting is further applied. Due to the insufficient number of participants in the elderly experimental group, we only used the data of the first four groups in the fitting. The relation between the flow rate and the bottleneck width $b$ fit the formula $\mathrm{J}_{e}=2.33 b-0.42, \mathrm{~J}_{y}=2.53 b-0.20$ and $\mathrm{J}_{m}=1.91 b-0.15$ for the elderly, young and mixed groups separately. The flow rate of the separate young group is the highest in these three groups. The mixture of the young and the elderly pedestrians diminish the flow rate significantly. As can be found in Fig. 2(a), the flow rate of the mixed group is lower than the young even the elderly group. One reason not to be overlooked is that the mixed group has more participants in each run than the separate elderly group. In addition, the relation slope of the mixed group is the smallest. It indicates that the mixture of pedestrians in different age limits the increase of the flow rate $\mathrm{J}$ with the exit width $b$. This is a reflection of the mixed effect of pedestrians with different ages. Fig. 2(b) shows the specific flow rate $\mathrm{J}_{S}$ which represents the traffic capacity of the pedestrians. The separate young group has the significant advantages for they have stronger movement ability and greater flexibility. The capacity of the mixed group is around $1.7 \mathrm{ped} /(\mathrm{m} \cdot \mathrm{s})$ which is lower than the separate young group even the elderly. In the mixed crowd, there are some phenomena that affect the traffic capacity, such as the modesty of the young to the old. Heterogeneity of age and movement ability in the population leads to a decrease in specific flow. This reminds us that in the study of pedestrian dynamics, we need to pay attention to the influence of the mixing effect of pedestrians with different compositions.

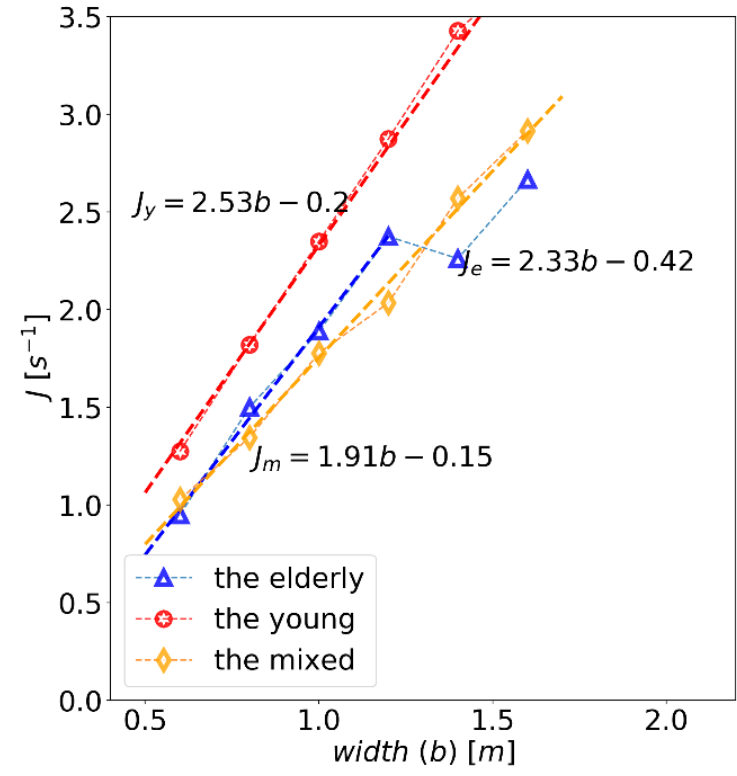

(a)

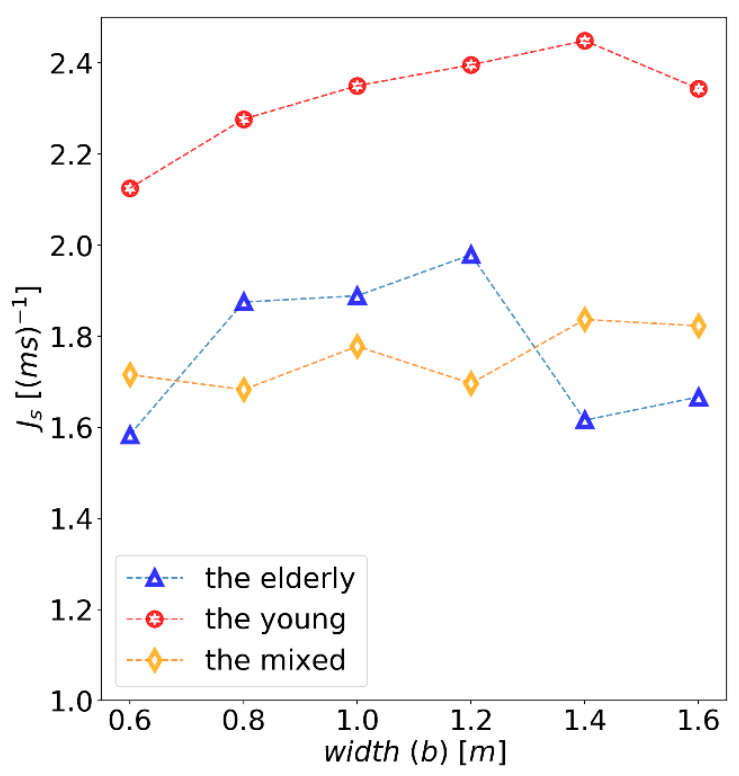

(b)

Figure 2 The flow rate (a) and the specific flow rate (b) against the exit width b of the separate elderly group, separate young group and the mixed group 


\subsection{Speed difference}

The speed of the elderly and the young in the mixed group are calculated separately to study the mixing effect furtherly. It can be found in the Fig. 3(a) that the average speeds of the young under different exit width $b$ are lower than that of the elderly. It was proved that the speed of the elderly is lower than that of the young people under the same density due to the decreased flexibility and movement ability of the elders [18]. However, the young pedestrians in the mixed group did not attempt to pass the elderly for safety considerations. They sacrifice their own speeds to maintain the movement synchronous with the old pedestrians. On the other hand, the young pedestrians keep a certain distance from the elderly in order to avoid collisions, so that the speed of the young is lower than that of the elderly in the mixed group. This is the effect of a mix of pedestrians with different ages which also can be reflected in the fundamental diagram. See Fig. 3(b). Where the measurement area is chosen as the axis $x$ from $3 \mathrm{~m}$ to $5 \mathrm{~m}$ and the $y$ axis from $0.75 \mathrm{~m}$ to $2.75 \mathrm{~m}$. The speed and density are calculated based on the Voronoi method [14] using the data from the steady stage excluding the start and the end stage. The smaller scatters show the calculated results every 10 frames and the larger scatters represent the average values. It can be observed that the average speed of the mixed group is between that of the separate young group and the separate elderly group. The high density of the mixed group distributes between $2.0 \mathrm{~m}^{-2}$ to $2.5 \mathrm{~m}^{-2}$ which is manifest lower than that of the separate young group. The average speed of the mixed group in this region has relatively large fluctuations. This is because that the young pedestrians avoiding collisions with the elderly limits the formation of higher densities. The heterogeneous composition of pedestrians in the mixed group caused the fluctuation of average velocity.

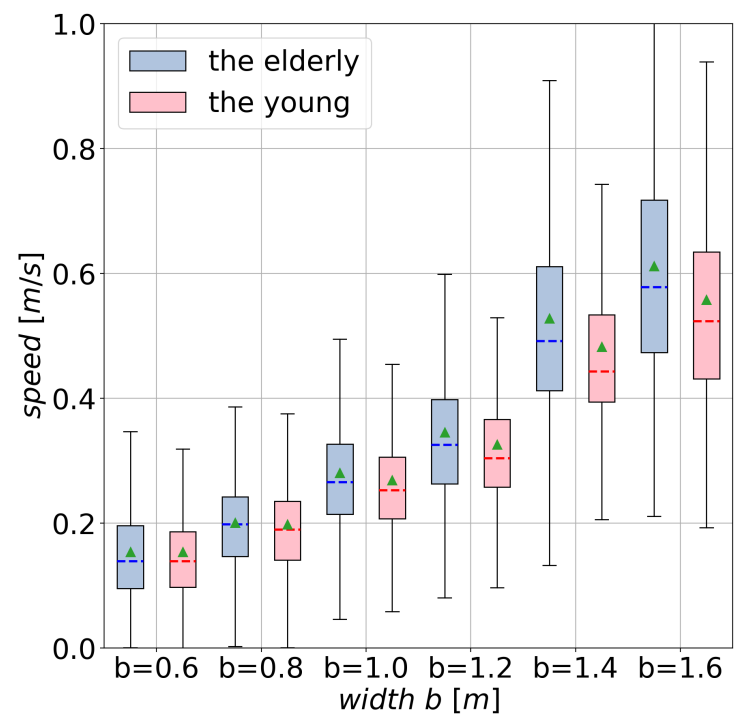

(a)

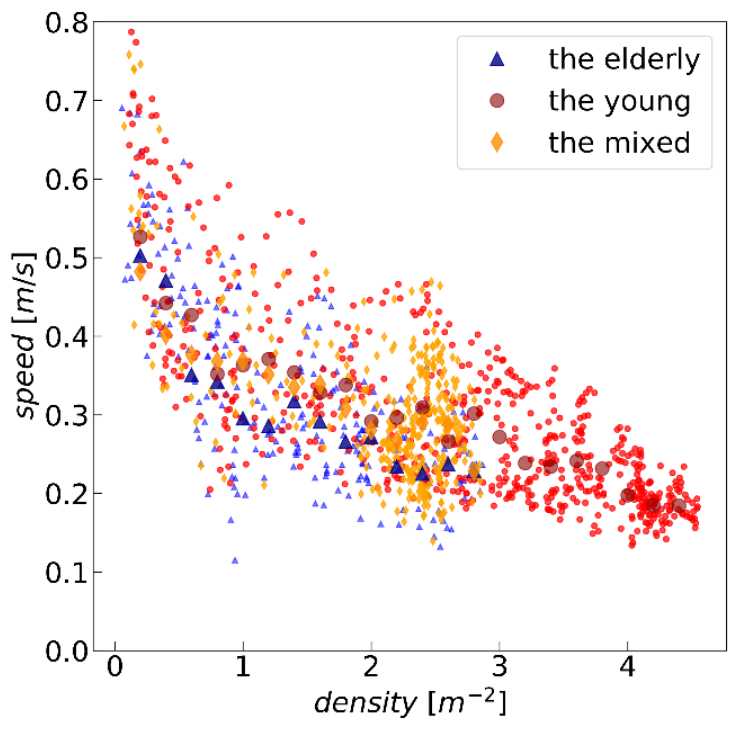

(b)

Figure 3 (a) The instantaneous speed of the elders and the young pedestrian in the mixed group. (b) The fundamental diagram of these three groups with different compositions 


\subsection{Density profiles}

As described in [19], the spatiotemporal profiles of the density spatial distributions can help to study the dynamics of the motion and the sensitivity of the integrated quantities to influencing factors. Consequently, the spatiotemporal profiles are studied for insights into the dynamic discrepancy of groups with different compositions. The densities integrated over the state stage in small regions $(0.1 \mathrm{~m} \times 0.1 \mathrm{~m})$ are calculated based on the Voronoi method and the profiles over the experimental areas are obtained. Fig. 4 shows the profiles for runs of $b=0.6$ and $1.0 \mathrm{~m}$ and $\mathrm{E}, \mathrm{Y}, \mathrm{M}$ represent the elderly, young and mixed groups respectively. Only data in the steady stage excluding the start and the end stages are used to reduce the fluctuation. For the distinct spatial distribution information, the Gaussian filtering procedure is used in the data filtering. The contour lines according to the change of density gradient are plotted to show the transitions of different density stages.

It is clear that the distributions of density are inhomogeneous over space for all of these three groups. While the density of the separate young group is the highest than the other groups under the same width $b$ for there is a greater degree of vitality and competition among young pedestrians. In addition, the shape of the pick-density around the exit can reflect the degree of competition of the crowd [20]. For instance, the arch distribution can be observed in the simulation with the social force model [21]. In our study, the distribution ranges and shapes of the high density for these three groups are different obviously. For the separate elderly group, the peak-density region displays an ellipse shape with a longer major axis. For the young group, it is similar to teardrop-shape distribution for the young which consists with the results in [9]. While the profiles of the mixed group present as long strips. It is because that the pedestrians with large age differences have greater distances and less competition for the sake of safety. The mixture of the young and elderly pedestrians dilutes the congested degree of the high-density areas in front of the bottleneck.

\subsection{Time headway and spatial distribution}

For further study of the movement properties of elderly pedestrians in the bottleneck flow with different compositions, the spatial and temporal characteristics are analysed in this section. Fig. 5(a) shows the time-space diagram of the mixed group under width $b=0.6 \mathrm{~m}$ to show the spatial-temporal characteristics qualitatively. Obvious gap in time and space can be found between the young and the elderly. This is due to the distance between the young and the old in the mixed group. Further, the time headway and the spatial distribution of different groups are calculated quantitively. In the same way, only data in the steady stage are used. It can be found from Fig. 5(b) that whether the elderly or the young pedestrians in the mixed group have longer time headway than them in the separate group. The separate elders have longer time headway than the separate young while the opposite is true for them in the mixed group. Which indicates that the young pedestrians consume time for avoiding collision with the old.

Fig. 6 shows the relative probability statistics of the spatial distribution for the pedestrian's nearest neighbour. The darker color represents the greater probability of the dis- 

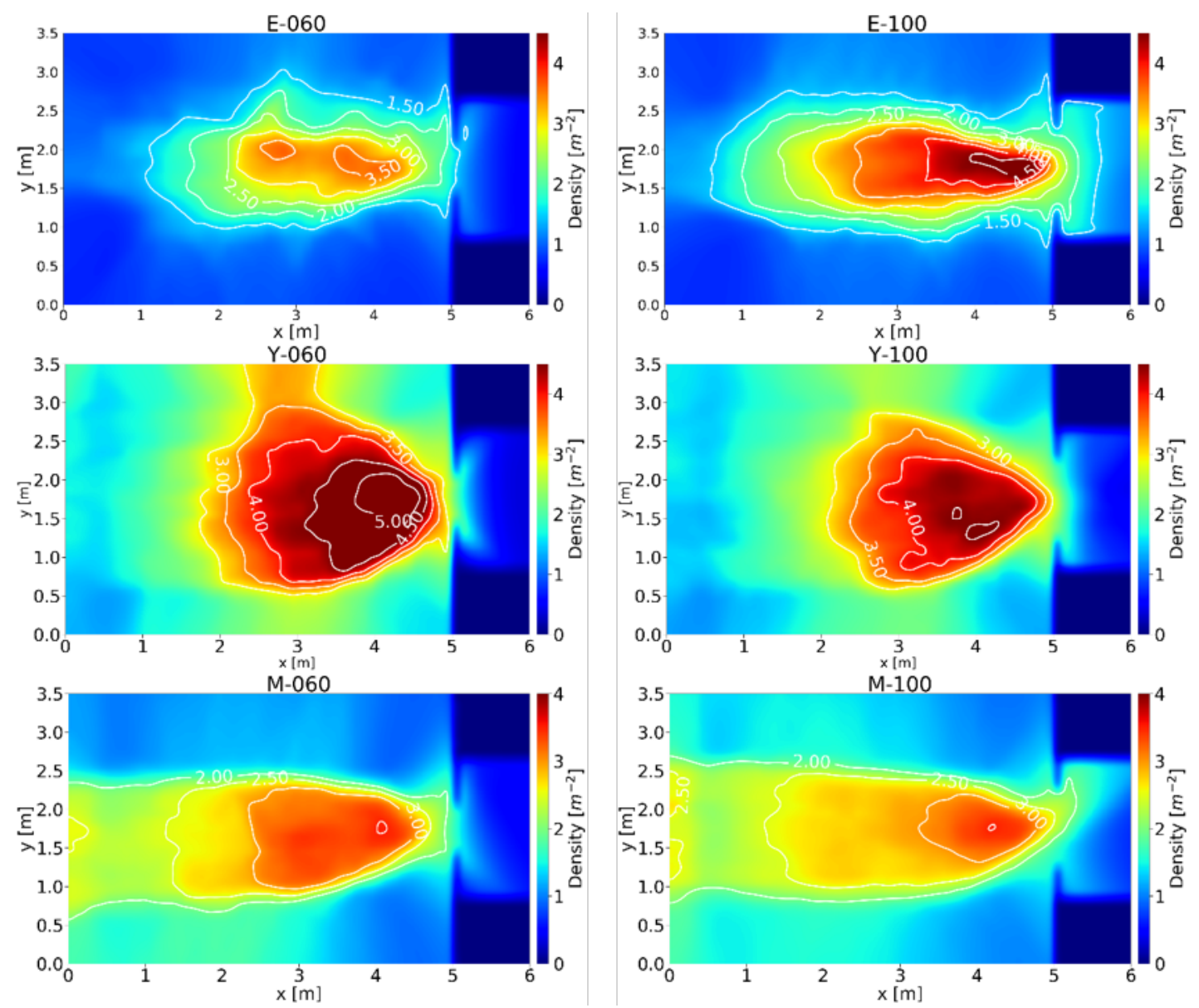

Figure 4 The high-density profiles of groups with different compositions in the run of $b=0.6$ and $1.0 \mathrm{~m}, \mathrm{E}$ is for the elderly group, $\mathrm{Y}$ is for the young group and $\mathrm{M}$ is for the mixed group

tribution. The spatial distributions of the separate and mixed group for the elderly and young have significant difference. In the separate groups, the spatial distribution of the nearest neighbour presents a uniform circle for both of the elderly and the young. The spatial distance between the elderly $(0.46 \pm 0.14 \mathrm{~m})$ is bigger than that between the young $(0.39 \pm 0.09 \mathrm{~m})$ because of the difference in number and density. While in the mixed group, there is no obvious difference between the distance of the elderly $(0.44 \pm 0.09 \mathrm{~m})$ and the young $(0.45 \pm 0.08 \mathrm{~m})$. The distributions of the nearest neighbour on the left and right side of the pedestrian are of higher probability than that in the front and rear. This indicates that old pedestrians are closer to the old and the young are closer to the young according to the way of line up.

\section{Conclusion}

Under the background of aging population, the movement properties of the elderly pedestrians in the bottleneck flow with different compositions were studied experimentally. 


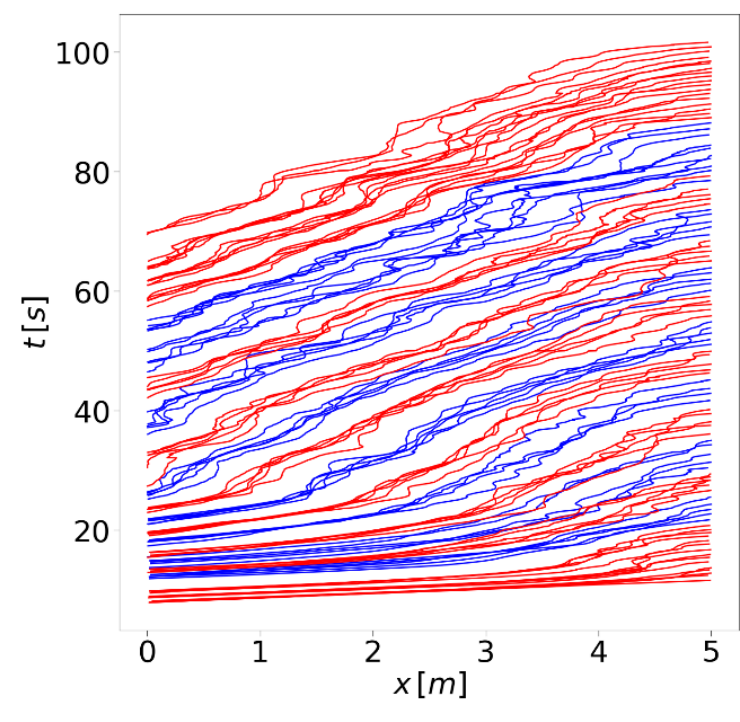

(a)

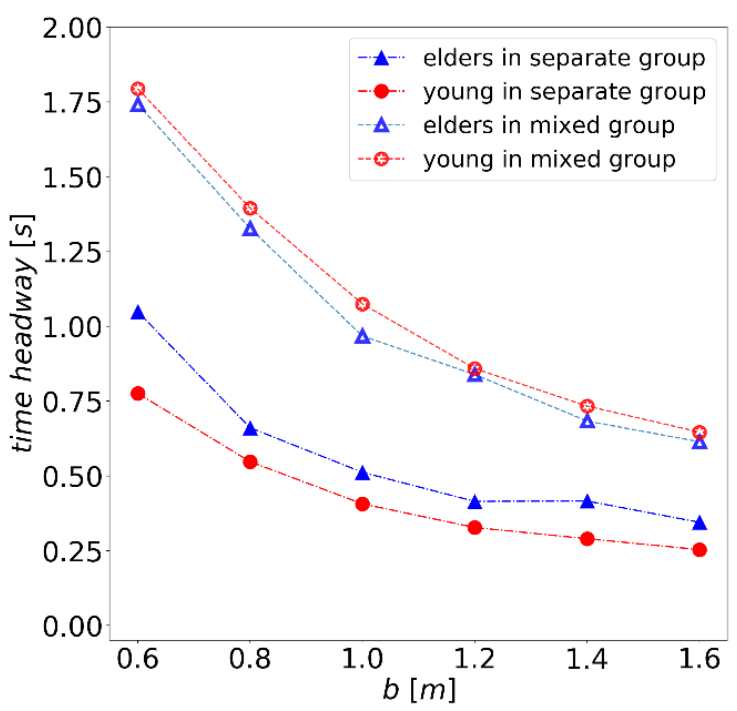

(b)

Figure 5 (a) The time-space diagram of the mixed group under exit width $b=0.8 \mathrm{~m}$, blue for the elderly and red for the young. (b) The time headway of the elders and young in the separate and mixed groups

Different compositions of the crowd were considered, including the elderly group, young group separately and the mixed group. Qualitative and quantitative analysis of the parameters, such as flow, velocity, density, the spatial and temporal characteristics, were carried out for the comprehensive understanding of the influence of the elderly pedestrians on the pedestrian and evacuation dynamic. Through the comparison of the relations between the flow rate, specific flow rate and the bottleneck width $b$, it can be found that the mixture of the elderly and the young in the mixed group makes the efficiency decrease significantly and even lower than that of the separate elderly group. Based on the linear fitting, the flow $J$ - width $b$ relations fit the formula $\mathrm{J}_{e}=2.33 b-0.42, \mathrm{~J}_{y}=2.53 b-0.20$ and $\mathrm{J}_{m}=1.91 b-0.15$ for the elderly, young and mixed groups separately. The mixed group has the smallest slope in the $J-b$ relation which indicates that the mixture of pedestrians of different age limits the increase of the flow rate $J$. The factors can be found from the analysis of speed, density and spatial-temporal characteristics.

In the mixed group, the young pedestrians sacrifice their speeds to keep synchronous with the elderly, which leads to the average speeds of the young pedestrians are lower than that of the elders. From the fundamental diagram, it can be observed that the density of the mixed group is lower than that of the separate young group. In addition, the high-density profiles of the mixed group present as long strips which indicates that the congestion degree in front of the exit is diluted. It is because that the young kept a certain distance from the elders for the sake of safety. From the temporal respect, whether the young or the elderly pedestrians in the mixed group have longer time lapse than in the separate groups when passing the exit. In the separate groups, the spatial distribution of the nearest neighbour presents a uniform circle. The spatial distance between the elderly $(0.46 \mathrm{~m})$ is 

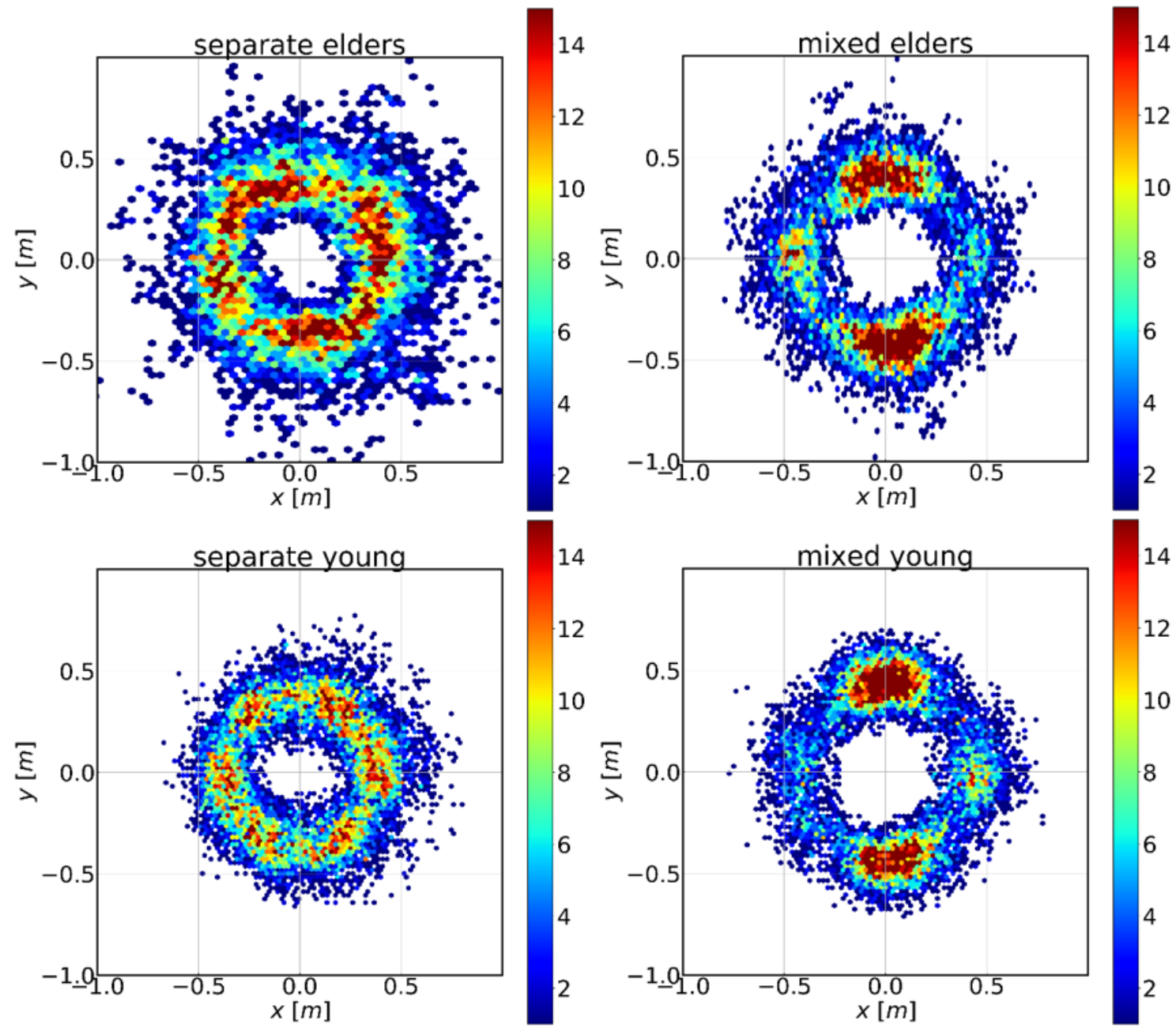

(a)

(b)

Figure 6 The spatial distribution of the nearest neighbour of the elders and the young in the separate group (a) and mixed group (b) 
bigger than that between the young $(0.39 \mathrm{~m})$. While in the mixed group, there is no obvious difference between the distance of the elderly $(0.44 \mathrm{~m})$ and the young $(0.45 \mathrm{~m})$. The distributions probabilities of the nearest neighbour on the left and right side are of higher for pedestrians of similar age are closer to each other.

The mixture of the elderly and the young pedestrians have significant influence on the pedestrian and evacuation dynamic. In the mixed flow with young and elderly pedestrians, the young slow down and keep certain distance with the elders for the sake of safety and avoiding collisions. It limits the speed of the young, reduce the density of the crowd and increase the time for pedestrians to pass the exit. Consequently, the mixture of the young and elders has a negative impact on the efficiency of pedestrian flow. The influence of the elderly pedestrians on the traffic flow are studied in this paper which is helpful for the modelling and management.

Acknowledgements The authors acknowledge the foundation support from the National Natural Science Foundation of China (Grant No. U1933105, 72001095, 71704168), from Anhui Provincial Natural Science Foundation (Grant No. 1808085MG217) and the Fundamental Research Funds for the Central Universities (Grant No. WK2320000040, WK2320000043).

\section{References}

[1] M. Haghani: Empirical methods in pedestrian, crowd and evacuation dynamics: Part I, experimental methods and emerging topics. Saf. Sci. 129, 104743 (2020), doi:10.1016/j.s.sci.2020.104743

[2] M. Haghani: Empirical methods in pedestrian, crowd and evacuation dynamics: Part II. Field methods and controversial topics. Saf. Sci. 129, 104760 (2020), doi:10.1016/j.ssci.2020.104760

[3] I. Tournier, A. Dommes, and V. Cavallo: Review of safety and mobility issues among older pedestrians. Accid. Anal. Prev., 91, 24-35 (2016), doi:10.1016/j.aap.2016.02.031

[4] X. Ren, J. Zhang, S. Cao, and W. Song: Experimental study on elderly pedestrians passing through bottlenecks. J. Stat. Mech. Theory Exp. 2019123204 (2019), doi:10.1088/1742-5468/ab4fdf

[5] S. Cao, J. Zhang, D. Salden, J. Ma, C. Shi, and R. Zhang: Pedestrian dynamics in single-file movement of crowd with different age compositions. Phys. Rev. E 94 1-11 (2016), doi:10.1103/PhysRevE.94.012312

[6] I. Zuriguel et al.: Clogging transition of many-particle systems flowing through bottlenecks. Sci. Rep. 4 1-8 (2014), doi:10.1038/srep 07324

[7] X. Shi, Z. Ye, N. Shiwakoti, D. Tang, and J. Lin: Examining effect of architectural adjustment on pedestrian crowd flow at bottleneck. Physica A 522 350-364 (2018), doi:10.1016/j.physa.2019.01.086 
[8] T. Kretz, A. Grünebohm, and M. Schreckenberg: Experimental study of pedestrian flow through a bottleneck. J. Stat. Mech. Theory Exp. 200610014 (2016), doi:10.1088/1742-5468/2006/10/P10014

[9] J. Liddle, A. Seyfried, W. Klingsch, T. Rupprecht, A. Schadschneider, and A. Winkens: An Experimental Study of Pedestrian Congestions: Influence of Bottleneck Width and Length. (2009) arXiv preprint arXiv: 0911.4350

[10] S. M. V. Gwynne, E. D. Kuligowski, J. Kratchman, and J. A. Milke: Questioning the linear relationship between doorway width and achievable flow rate. Fire Saf. J. 44 80-87 (2009), doi:10.1016/j.firesaf.2008.03.010

[11] X. Ren, J. Zhang, S. Cao, and W. Song: Experimental study on elderly pedestrians passing through bottlenecks. J. Stat. Mech. Theory Exp. 2019123204 (2019), doi:10.1088/1742-5468/ab4fdf

[12] E. Kuligowski, R. Peacock, E. Wiess, and B. Hoskins: Stair evacuation of older adults and people with mobility impairments. Fire Saf. J. vol.62 230-237 (2013), doi:10.1016/j.firesaf.2013.09.027

[13] L. D. Vanumu, K. Ramachandra Rao, and G. Tiwari: Fundamental diagrams of pedestrian flow characteristics: A review. Eur. Transp. Res. Rev. 9 1-13 (2017), doi:10.1007/s12544-017-0264-6

[14] B. Steffen, A. Seyfried: Methods for measuring pedestrian density, flow, speed and direction with minimal scatter. Physica A 389 1902-1910 (2010), doi:10.1016/j.physa.2009.12.015

[15] A. Garcimartín, I. Zuriguel, J. M. Pastor, C. Martín-Gómez, and D. R. Parisi: Experimental evidence of the 'faster is slower' effect. Transp. Res. Procedia 2 760-767 (2014), doi:10.1016/j.trpro.2014.09.085

[16] J. Porzycki, M. Mycek, R. Lubas, and J. Waos: Pedestrian spatial self-organization according to its nearest neighbor position. Transp. Res. Procedia 2 201-206 (2014), doi:10.1016/j.trpro.2014.09.033

[17] M. Boltes, A. Seyfried, B. Steffen, and A. Schadschneider: Automatic Extraction of Pedestrian Trajectories from Video Recordings. Pedestr. Evacuation Dyn. 2008 43-54 (2010), doi:10.1007/978-3-642-04504-2

[18] X. Ren, J. Zhang, W. Song, and C. Shuchao: The fundamental diagrams of elderly pedestrian flow in straight corridors under different densities. J. Stat. Mech. Theory Exp. 2019023403 (2019), doi : $10.1088 / 1742-5468$ / a afa 7b

[19] J. Zhang and A. Seyfried: Quantification of bottleneck effects for different types of facilities. Transportation Research Procedia 2 51-59 (2014), doi:10.1016/j.trpro.2014.09.008 
[20] M. Haghani, M. Sarvi, and Z. Shahhoseini: Evacuation behaviour of crowds under high and low levels of urgency: Experiments of reaction time, exit choice and exit-choice adaptation. Saf. Sci. 126104679 (2020), doi:10.1016/j.ssci.2020.104679

[21] D. Helbing, I. Farkas, and T. Vicsek: Simulating dynamical features of escape panic. Nature 407 487-490 (2000), doi: $10.1038 / 35035023$ 